\title{
IMPLEMENTATION OF A NATIONAL ELECTRONIC REPORTING SYSTEM IN LITHUANIA
}

\author{
M Domeika (marius.domeika@medsci.uu.se) ${ }^{1,2}$, G Kligys $^{3}, 0$ Ivanauskiene ${ }^{3}, \mathrm{~J}$ Mereckiene ${ }^{4}$, V Bakasenas ${ }^{5}$, B Morkunas $^{6}$, \\ D Berescianskis ${ }^{7}, \mathrm{~T} \mathrm{Wahl}^{8}$, K Stenqvist ${ }^{9,10}$ \\ 1. Department of Medical Sciences, Uppsala University, Uppsala, Sweden \\ 2. East Europe Committee of the Swedish Health Community, Stockholm, Sweden \\ 3. Kaunas Public Health Center, Kaunas, Lithuania \\ 4. Vilnius Public Health Center, Lithuania \\ 5. The State Public Health Service (SPHS) under the Ministry of Health, Lithuania \\ 6. Lithuanian Center for Communicable Diseases Prevention and Control, Lithuania \\ 7. Computer technology company "Compidea", Vilnius, Lithuania \\ 8. Department of Communicable Disease Control and Prevention, Stockholm County, Sweden \\ 9. Hivprevention i Västra Götaland, Region Västra Götaland, Sweden \\ 10. Department of Social Medicine, Sahlgrenska Academy, University of Gothenburg, Sweden
}

Electronic reporting systems improve the quality and timeliness of the surveillance of communicable diseases. The aim of this paper is to present the process of the implementation and introduction of an electronic reporting system for the surveillance of communicable diseases in Lithuania. The project which started in 2002 was performed in collaboration between Lithuania and Sweden and was facilitated by the parallel process of adapting the surveillance system to European Union (EU) standards. The Lotusbased software, SmittAdm, was acquired from the Department of Communicable Diseases Control and Prevention of Stockholm County in Sweden and adopted for Lithuania, resulting in the Lithuanian software, ULISAS. A major advantage of this program for Lithuania was the possibility to work offline. The project was initiated in the two largest counties in Lithuania where ULISAS had been installed and put in use by January 2005. The introduction was gradual, the national level was connected to the system during late 2005, and all remaining counties were included during 2006 and 2007. The reporting system remains to be evaluated concerning timeliness and completeness of the surveillance. Further development is needed, for example the inclusion of all physicians and laboratories and an alert system for outbreaks. The introduction of this case-based, timely electronic reporting system in Lithuania allows better reporting of data to the European Centre for Disease Prevention and Control (ECDC) and the World Health Organization (WHO) compared to the former reporting system with paper-based, aggregated data.

\section{Introduction}

Well-functioning surveillance systems for communicable diseases are fundamental in providing information needed to take appropriate and timely measures. Studies from countries with electronic reporting systems show that such systems improve the quality and timeliness of the surveillance [1-4]. In addition, there is need for an integrated European surveillance system so that the epidemiology of communicable diseases can be compared between countries and early warning systems can function in an international perspective. The present project was initiated in order to improve the Lithuanian system for the surveillance of communicable diseases which hitherto had been time consuming to administer and only paper-based, aggregated data had been reported to the national level. Duplication of data was also a problem since aggregated data was sent in parallel from both local and county level to the national centre. During the project period, Lithuania joined the European Union (EU). Consequently, the Lithuanian law on communicable diseases was adapted to the EU standards [5]. The main goal of this paper is to present the introduction process of the electronic surveillance system for communicable diseases ULISAS in Lithuania, a project that was performed with financial support and expertise from Sweden.

\section{Materials and methods \\ Organisation of the surveillance of communicable diseases in Lithuania}

Lithuania has 3.4 million inhabitants and is organised in ten counties, each with one Public Health Centre (PHC). Each county has one or more Territorial Public Health Centers (TPHC), altogether 36 in the country. The PHC has an overall responsibility for the surveillance of communicable disease within the county [6]. Notifiable diseases are reported from the PHC to the Lithuanian Centre for Communicable Diseases Prevention and Control (CCDPC) as shown in Figure 1.

Physicians from a total of 1,257 primary care centres, hospitals and polyclinics and 21 laboratories report notifiable diseases by post, fax or e-mail to the TPHC and the PHC within 72 hours according to the rules and regulations. In unusual situations, for example the occurrence of plague and yellow fever, notifications should be sent by post, fax or e-mail, within 12 hours. The clinical notifications contain full patient identity and a unique personal identification number which is issued to all Lithuanian residents. 
Exceptions are the sexually transmitted infections (STI) and cases of human immunodeficiency virus (HIV), which are reported with a specially designed code, so that the personal identity is not revealed. The number of clinical notifications amounts to about 55,000 per year in the whole country. The epidemiologic investigations of individual cases and outbreaks are performed by epidemiologists at the TPHC and the PHC and reported on standardised paper forms to the CCDPC. For STI and HIV, physicians perform the epidemiological investigation and report weekly to the TPHC and $\mathrm{PHC}$. Prior to the development of the electronic reporting system, aggregated data on 82 notifiable diseases collected at the TPHC and the PHC had been summarised using a standard statistical form at the end of every month and sent to the CCDPC in paper format. In addition, aggregated data had been reported yearly to the CCDPC using 17 different statistical forms.

\section{Organisation of the surveillance of communicable diseases in Sweden}

Notifiable diseases are reported by physicians to the County Medical Officer (CMO) at the county level and to the Swedish Institute for Infectious Disease Control (Smittskyddsinstitutet, SMI) at the national level. The CMO has an overall responsibility for the surveillance in his or her county. A national electronic surveillance system, SmiNet-1, had been operational from 1997 and in use by 16 of 21 counties since the beginning of the 2000s [3]. With this system, notifications were mainly sent in paper format to the $\mathrm{CMO}$ and manually entered at the county level, with the exception of the infectious diseases clinics and laboratories which were connected to the electronic reporting system. After a technical revision of SmiNet-1 in 2001, a new web-based system, SmiNet-2, was developed and implemented in 2004 [1]. Parallel to this, the Department of Communicable Diseases Control and Prevention at the county of Stockholm developed a program for electronic reporting, SmittAdm, which has been in use from 1998. This

\section{F I G U R E 1}

Data flow in the Lithuanian national reporting system of communicable diseases before and after the introduction of ULISAS electronic system

Data flow before introduction of ULISAS

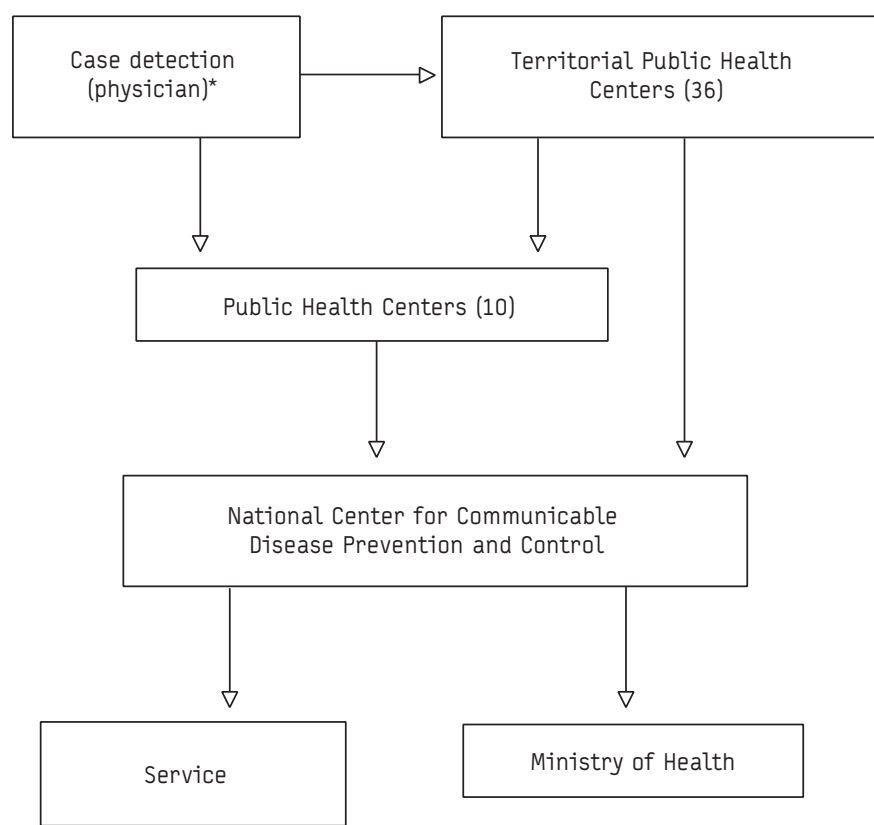

$\longrightarrow$ Aggregated data; monthly by e-mail

$\longrightarrow$ Indivudual data, daily, on-line

Ongoing introduction of the daily, on-line reporting, but temporarily both daily and aggregated data is reported
Data flow after introduction of ULISAS

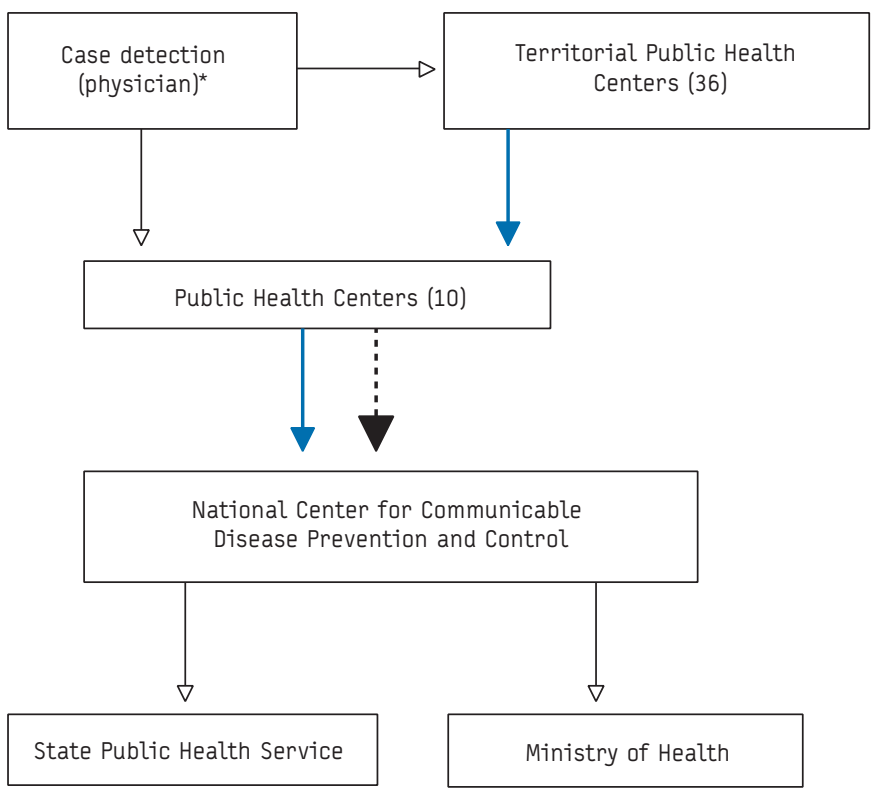

${ }^{\star}$ Case data - notification by phone, fax, mail and e-mail according to the legal requirements 
program supplemented SmiNet-1 with functions for administrative and judicial tasks which according to the Swedish Communicable Diseases Act are performed at the county level. SmittAdm and SmiNet-1, both being built in Lotus Notes, were easily connected. The staff at the county level used SmiNet-1 only for submitting notifications to the national level; data was then replicated to SmittAdm for further regional work and analysis. SmittAdm was also used by the second largest region, Västra Götaland, and three other counties.

\section{Project organisation}

The project was initiated in 2002 by the Swedish Institute for Infectious Disease Control (SMI) and the PHC in Kaunas with financial support from the East Europe Committee of the Swedish Health Care Community (SEEC).

The project manager (of Lithuanian origin, which facilitated communication) was based at SMI and a coordinating study group was formed in Lithuania consisting of epidemiologists from the national level and the counties of Kaunas and Vilnius, and an external IT specialist. This group defined the requirements for an electronic reporting system in Lithuania. Such a system should enable timely reporting of individual data to the county and national level, ascertain a uniform quality of notifications for the whole

\section{F I G U R E 2}

Electronic reporting system of communicable diseases ULISAS in Lithuania, the county level

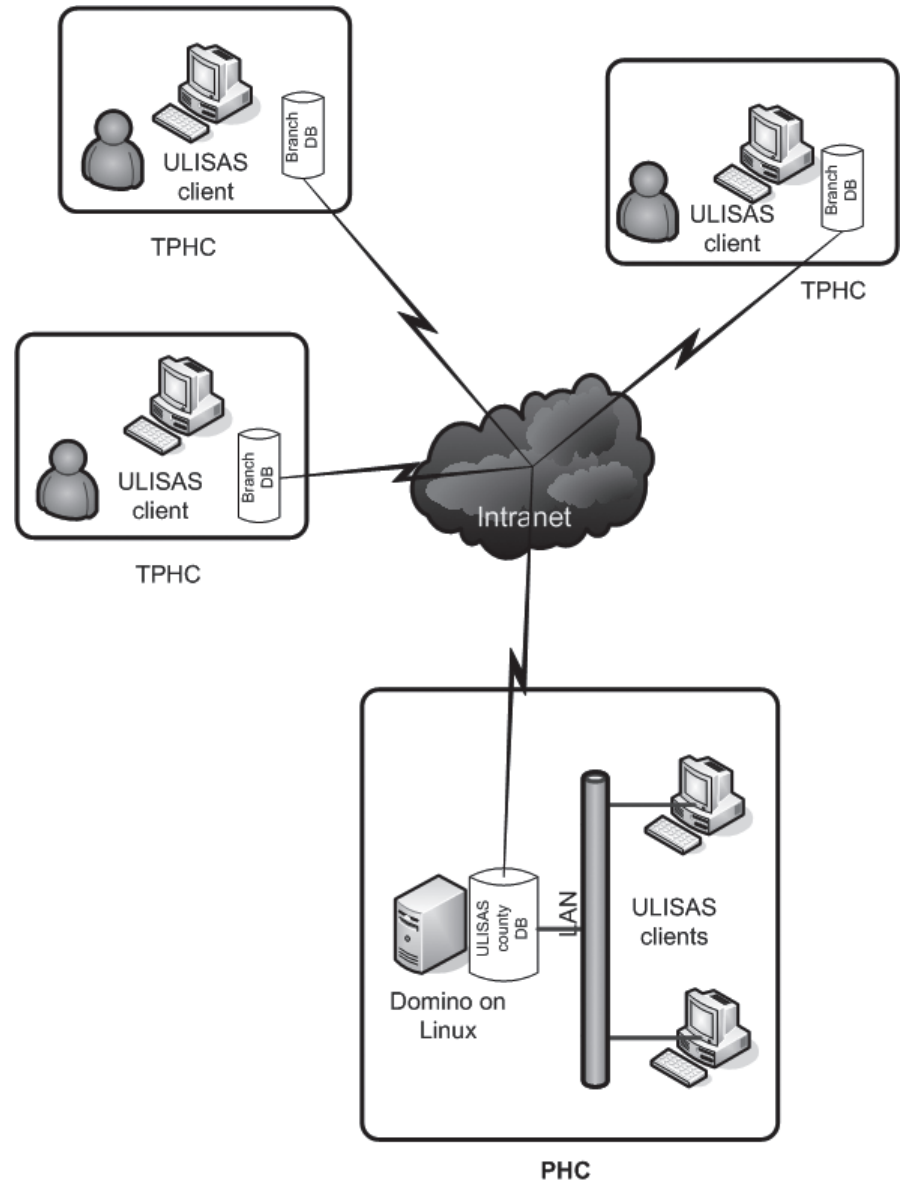

country, and support the integration of laboratory and clinical notifications. Due to limited access to the internet the system had to allow working offline at the TPHC and the PHC. Since the project was not fully financed from the beginning, extra costs for staff were to be avoided. The study group was responsible for: 1 ) revising the surveillance procedures and the list of notifiable diseases according to the EU requirements, 2) creating adequate epidemiological forms for notifiable diseases, 3) studying the present Swedish electronic surveillance systems and participating in the process of developing and adapting the software for Lithuania, 4) establishing an action plan for the implementation of the system in the Lithuanian organisation, 5) assuring the provision of hardware and software for participating units, and 6) organising training for users at all levels.

\section{Project sites}

The overall goal was to include all counties and the national level in the project. The plan was to start the project at the PHC of Kaunas and Vilnius, counties with the largest populations in Lithuania. The authorities in Kaunas and Vilnius were motivated to introduce an electronic surveillance system; they identified its potential to reduce their work load and to improve not only the surveillance at the national, but also at the county level. The next target would be the national level, the CCDPC, followed by the remaining eight counties in Lithuania. The reason why this step by step approach had to be taken was that the resources were limited at the national level, not allowing the CCDPC to be involved from the start, and that the project at this point lacked financing for the whole country.

\section{Results}

\section{Revision of notifiable diseases and notification forms}

The number of diseases notifiable in Lithuania was revised and reduced from 82 to 76 . The revisions were made in accordance with the Commission Decision No 2003/542/EC [7]. The lists of notifiable diseases and microorganisms were regulated by law by the Ministry of Health of the Republic of Lithuania in May 2004 and January 2005 respectively. New epidemiological forms were elaborated for nine groups of diseases and defined by the Director of the State Public Health Service under the Ministry of Health in June 2004 [9].

\section{Selection of electronic reporting system and development of the} software

Since the Lithuanian authorities wanted to see the result at county level before a central server was to be established, it was important to focus on the needs at the PHC level. SmiNet-1 and SmittAdm were both suitable in the respect that they allowed working offline, which was necessary since the internet was not sufficiently available for the majority of the PHC and TPHC. An advantage of SmittAdm was that this program, contrary to SmiNet-1, allowed integration of patient records and notes on contact tracing and outbreaks, facilitating work at the county level. Thus SmittAdm was chosen as the model for the Lithuanian software; a contributing factor for the choice was that SmittAdm was easily and quickly accessible to buy from the county of Stockholm. At the time SmiNet-2 had not been implemented in Sweden yet and would not be suitable since it was web-based [1].

\section{Software and hardware}

In 2004, SmittAdm was acquired from the county of Stockholm and translated into Lithuanian. Subsequently, a new program, the System for Data Collection and Analysis of Communicable Diseases 
(ULISAS), very similar to SmittAdm, was created in collaboration with an IT company, COMPIDEA. ULISAS uses the IBM Lotus Domino and Lotus Script and Java programming languages. The minimal requirement for ULISAS servers was any commercially available server with Pentium 2GHz, 2GB RAM, 2 HDD and 36 GB each, running Notes Domino 6.5 and higher (currently 8.0.2) on Linux or Windows servers and for workstations simple commercially available Windows computers with Lotus Notes 6.5 and higher. At the national level the requirements for the DB2 Lotus Domino server was $3 \mathrm{GHz}$, 4GB RAM, 300GB HDD.

\section{Databases and architecture of the system}

The ULISAS database is built in Lotus Notes. The staff from COMPIDEA monitors all activities and provides continuous support to users at the TPHC and PHC. The dataflow of the electronic reporting system is shown in Figure 1 . The electronic reporting system with workstations and servers at the county and the national levels is shown in Figures 2 and 3.
The TPHC: There is usually one Lotus Notes client per TPHC. Data is regularly exchanged between the PHC and the TPHC by replication so that the TPHC client can work offline if necessary. If more data entry places are needed, it is possible to install extra, standalone Lotus Notes clients which also replicate to the PHC database. The main three physical .nfs files contain patient records, notifications and staff activity records. The number of fields with defined entry values varies between 33 and 54 between the different records, seven fields are obligatory. Data quality is assured by validation during data entry against a set of validity rules. Further notes concerning a patient or an investigation can be entered in commentary fields. All case records related to one individual over time can be linked to one another. Data is transferred to the county server automatically every hour or manually by the operator at any time.

The PHC: Several Lotus Notes clients or work units are included in a LAN with one Lotus Domino server. The county server keeps records from all TPHC clients in the county, backup and a

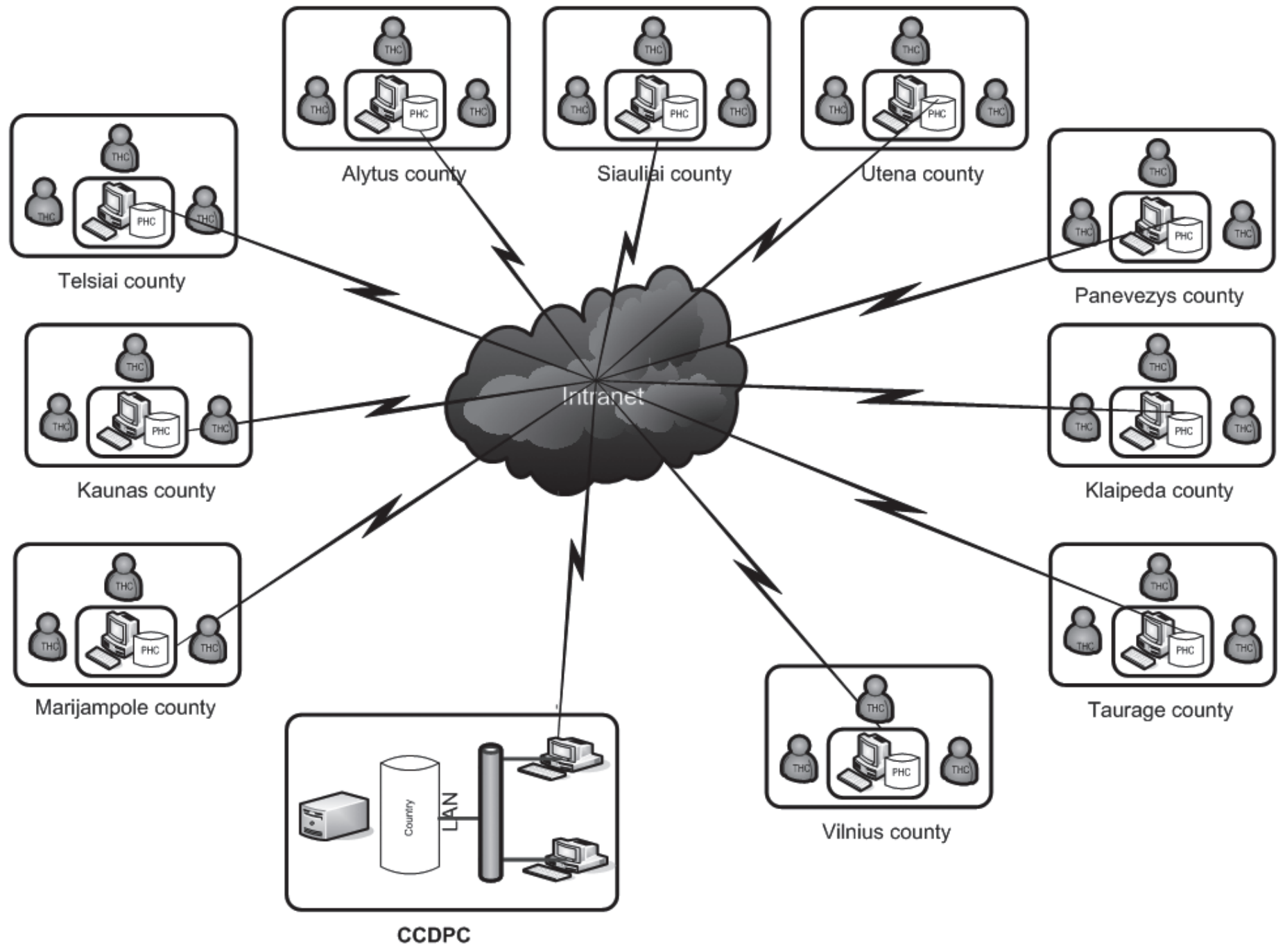


historical archive. Backup data can be transferred to an external USB HDD 300GB, allowing space to be maintained at the server. Data is regularly exchanged between the PHC and the CCDPC by replication, and when the internet is inaccessible or slow, the PHC server repeats replication in the following communication interval.

Laboratories: So far only one laboratory in the county of Kaunas has joined the system. Data is entered manually and the Lotus Notes workstation replicates data to the PHC and then to the CCDPC. It is possible to link a laboratory notification to the corresponding clinical notification.

The CCDPC: Several work units in the LAN at the national level are connected to a Lotus Domino server. Data from the databases in the ten counties is replicated to the central database; the CCDPC has an additional relational database based on DB2 version 8.2 in order to integrate the databases from each county. Defined fields from the records are exported to DB2 for further statistical analysis in SPSS. All data is archived in the central server.

\section{Present organisation of the surveillance of communicable diseases in Lithuania}

Case-based data is reported continuously via the electronic reporting system from the $\mathrm{TPHC}$ to the $\mathrm{PHC}$ and further to the CCDPC as demonstrated in Figure 1. Since the national level has not yet developed a system for analysis of the electronic reports, the old and new reporting systems still work in parallel. However, a change has been made so that the TPHC submits reports to the $\mathrm{PHC}$ only, which means that duplicates are avoided.

\section{Implementation process}

During the preparatory phase, 2002-2004, the main task of the project leader was to mediate collaboration between specialists from Kaunas PHC, the SMI and the Department of Communicable Diseases Control and Prevention, Stockholm County in Sweden. It was also important to stimulate a dialogue between the pioneering counties and the national Lithuanian authorities concerning the full implementation of ULISAS. By January 2005, ULISAS had been implemented in the counties of Kaunas and Vilnius with two servers and nineteen workstations at the PHC and the TPHC. The IT company trained the senior county epidemiologists who thereafter trained the remaining staff. During 2005, export functions for statistical analysis were developed. Later during 2005, the central server at the national level was installed and connected to the existing servers at the county levels. By 2006, a further six counties with 35 workstations were connected to the reporting system. The last two counties joined the system in 2007 when a total of 70 workstations were functioning. Personnel were trained as soon as their local working stations were installed and all throughout the project. Contracts for long term distance maintenance of the software and the hardware were signed. During 2009, servers are to be installed at the national level allowing all local servers to be connected into one national system.

\section{Further financial and political support}

In April 2004, the Director of the State Public Health Care Service under the Ministry of Health issued an order to initiate "The Study for the Implementation of the Computerised Program for Epidemiological Surveillance of Communicable Diseases at Kaunas and Vilnius Public Health Centres" [9]. This resulted in the provision of hardware for the CCDPC and a further six counties. The last two counties were included with financial support from the Swedish- Lithuanian project. The full integration of the reporting system with the national level will be supported by a LithuanianNorwegian project during 2009. The fact that costs for staff at the $\mathrm{TPHC}$ and PHC levels was reduced facilitated the financing of the project. This can be exemplified by the county of Kaunas where the implementation of the new system with centralised organisation of the work process resulted in a reduction of costs for statisticians by 75 percent and for IT support by 85 percent. Staff members at the TPHC and the PHC were made redundant. The IT company use remote control in combination with hotline support, server administration and back-ups are managed centrally. The total costs for the development of the electronic reporting system is estimated to 60,000 EURO, the cost for hardware not being included since existing hardware was used. The yearly maintenance and support of the system amount to $12,000-15,000$ EURO.

\section{Data output}

The national analysis is still based on monthly aggregated data from the ten counties and the reports to the ECDC the WHO have not yet been changed. The PHC of Kaunas developed a website during the project where statistics in the form of tables, graphs and maps were presented [10]. A corresponding website will be accessible at the CCDPC after the full integration of the national level during 2009.

\section{Data security}

Each individual user at the TPHC, PHC and CCDPC is given a Lotus Notes ID file protected by a password. Users have varying degree of access rights to the system depending on his or her function. All data in the database is encrypted and all data is transferred through encrypted channels. A governmental agency provides internet access for the system. A control system for further quality assurance is developed by the IT company during 20082009.

\section{Discussion}

In this paper we outline the structure and implementation of ULISAS, a new comprehensive electronic reporting system for the surveillance of communicable diseases in Lithuania. The process, which started in 2000, has led to a change from paper-based aggregated monthly data at the county and national level to a timely case-based electronic reporting system. Parallel to this, the number of notifiable diseases was standardised according to the EU case definitions. The initiative and establishment of ULISAS was a joint venture between Lithuania and Sweden, the communication between the two counties and Sweden and financing through the SEEC being of vital importance [10]. Political engagement and further financial support was facilitated by the new Lithuanian legislation in 2001 on communicable diseases and the EU directives concerning notifiable communicable diseases [5].

The organisation of the surveillance of communicable diseases in Lithuania and Sweden are similar, the main difference is the existence in Lithuania, but not in Sweden, of local public health centres, TPHC. Epidemiologists at the TPHC perform epidemiological investigations on patients who have been reported with notifiable communicable diseases by the physicians $[6,8]$. For diseases belonging to the STI group, the same as in Sweden, a physician is responsible for the epidemiological investigation [12]. The main objective of the planned cooperation between the two countries was that Lithuania should take advantage of the Swedish experiences concerning electronic reporting systems. At the start of the project, Sweden had a national electronic reporting system in use, SmiNet-1, built in Lotus Notes. Since SmiNet-1 
did not have functions for administrative notes and records on patients and contact tracing a complementary program, SmittAdm, had been developed by the county of Stockholm. The Lithuanian project group chose SmittAdm as the prototype for the Lithuanian reporting system because it met the requirements at both county and national level, most importantly the possibility to work offline. A disadvantage with the choice of a Lotus Notes based program lay in creating export functions for statistical analysis and reporting, i.e. tasks that are not primarily performed with Lotus Notes. The new Swedish electronic reporting system, SmiNet-2, was under development during the study period but was not an alternative for Lithuania since it was web-based.

The implementation of the system in Lithuania started in 2004 and by 2007 the whole country had been covered with a total of 70 workstations and trained staff at the county level. The bottom-up policy with the work process starting at the county level was crucial for the completion of the project. The two counties with the largest populations initiated the project from the Lithuanian side and were able to develop the program from the requirements at the county level. The staff in these counties with the heaviest workload was motivated to change to an electronic reporting system. In addition, they were able to initiate the present project since they had access to hardware through previous state-supported programs. The national level had not been involved until late 2005 after the system had been established in the two pilot counties. This was in accordance with the initial plan and due to the fact that resources were lacking at the national level, and that it had to be proven that the system worked before the national level was connected. Financing was a risk factor in this project, since resources were limited and financial support was granted step by step. This explains why the national level has still not been fully integrated in the project, still lacking instruments for the analysis and data output. For comparison, the Swedish reporting system SmiNet-1, which was in use between 1997 and 2004, was not implemented in all counties [3]. This may be due to the fact that the Swedish organisation is decentralised and that some counties had developed their own tailor-made systems.

ULISAS needs to be further developed. Physician and laboratory notifications from the whole country should join the system in the future and algorithms for the detection of outbreaks should be elaborated. When access to the internet is stable at all levels a web application may be developed so that ULISAS can be extended to private clinics and physicians. The future work and development of ULISAS will be supported by the National Public Health Strategy Implementation Plan in 2006- 2013 [13].

The impact of ULISAS on the surveillance of communicable diseases in Lithuania remains to be evaluated. When the national level has joined to full extent it will be possible to leave the old system and to analyse the data from the new case-based electronic reporting system, to present data on the national website and to adapt better to the European surveillance system administered by ECDC and reporting of data to the WHO. According to the ECDC there is a wide variability in the design and effectiveness of the surveillance systems between countries [14]. With ULISAS, Lithuania has developed an important tool for further adaption to the EU directives.

\section{Aknowledgements}

The present study was supported by a grant from the East Europe Committee of the Swedish Health Care Community, SIDA. We would also express our gratitude to the head of the Department of Communicable Disease Control and Prevention, Uppsala County - Staffan Sylvan and Stockholm County - Inger Berggren Palme, as well as the former head of the Department of Epidemiology of the Swedish Institute for Infectious Disease Control (Smittskyddsinstitutet, SMI) Johan Giesecke, for consultations and support.

\section{References}

1. Rolfhamre P, Janson A, Arneborn M, Ekdahl K. SmiNet-2: Description of an internet-based surveillance system for communicable diseases in Sweden. Euro Surveill. 2006;11(5):pii=626. Available from: http://www.eurosurveillance. org/ViewArticle. aspx?ArticleId $=626$

2. National Electronic Disease Surveillance System (NEDSS): a standards-based approach to connect public health and clinical medicine. J Public Health Manag Pract 2001;7(6):43-50.

3. Jansson A, Arneborn M, Ekdahl K. Sensitivity of the Swedish statutory surveillance system for communicable diseases 1998-2002, assessed by the capture-recapture method. Epidemiol Infect. 2005;133(3):401-407.

4. Ward M, Brandsema P, van Straten E, Bosman A. Electronic reporting improves timeliness and completeness of infectious disease notification, The Netherlands, 2003. Euro Surveill. 2005;10(1):pii=513. Available from: http:// www.eurosurveillance.org/ViewArticle.aspx?ArticleId=513

5. Seimas of the Republic of Lithuania. Law on the Amendment to the Law on Prevention and Control of Communicable Diseases in Humans, Nr. X-1149, 2007-05-24, Žin., 2007, Nr. 64-2454 (2007-06-09). Available from: http://www3. lrs.lt/pls/inter3/dokpaieska.showdoc_bin?p_id=157347

6. Kligys G. Epidemiological surveillance and control system of communicable diseases: management principles of sexually transmitted infections. Materials of the Conclusive conference of the Lithuanian-Swedish project "Improvement of the prevention and control of sexually transmitted infections in Lithuania". 26 May 2005, Vilnius, Lithuania. p. 45-48. Available from: http://www.medsci. uu.se/klinbakt/stigup/Interesting\%20from\%20conferences/05\%2005\%2026\%20 Kaunas\%20conf/Angliskas_visas.pdf

7. Commission Decision of 17 July 2003 amending Decision 2000/96/EC as regards the operation of dedicated surveillance networks (notified under document number C(2003) 2522) (Text with EEA relevance). 2003/542/EC. Official Journal of the European Union, 24.07.2003. L 185/55-58. Available from: http://eur-lex. europa.eu/LexUriServ/LexUriServ.do?uri=0J:L:2003:185:0055:0058:EN:PDF

8. Ministry of Health: Statutory epidemiological registration and reporting order of the epidemiological registration subjects 2002:673; 2004:V-397; 2005:V-38. Available from: http://www.sam.lt/lt/main/teisine_informacija/ ministro_isakymai?id=24806

9. Public Health Service (SPHS) under the Ministry of Health. Available from: http://www.vvspt.lt/teises.php?p=ta_visakymai

10. Kaunas Public Health Centre. Available from: http://kaunas.vvspt.lt

11. Ivanauskiene 0 . The surveillance of communicable diseases in Lithuania after implementing the computerised epidemiological data management program at Kaunas and Vilnius Public Health Centers. Materials of the Conclusive conference of the Lithuanian-Swedish project "Improvement of the prevention and control of sexually transmitted infections in Lithuania". 26 May, 2005 Vilnius, Lithuania. P. 48-52. Available from: http://www.medsci.uu.se/klinbakt/ stigup/Interesting\%20from\%20conferences/05\%2005\%2026\%20Kaunas\%20conf/ Angliskas_visas.pdf

12. Ministry of Health of Lithuania. The epidemiological surveillance procedures of sexually transmitted infections, HIV transmittance and HIV disease in primary health care centres and public health institutions, 2003: V-117. Available from: http://www.sam.lt/lt/main/teisine informacija/ministro isakymai $?$ id $=24856$

13. Government of the Republic of Lithuania: National public health strategy implementation plan of 2006-2013. Available from: http://www3.lrs.lt/pls/ inter3/dokpaieska.showdoc_bin?p_id=292274

14 Amato-Gauci A, Ammon A. ECDC to launch first report on communicable diseases epidemiology in the European Union. Euro Surveill. 2007;12(23):pii=3213. Available from: http://www.eurosurveillance.org/ ViewArticle.aspx?ArticleId $=3213$

This article was published on 2 April 2009

Citation style for this article: Domeika M, Kligys G, Ivanauskiene 0, Mereckiene J, Bakasenas V, Morkunas B, Berescianskis D, Wahl T, Stenqvist K. Implementation of national electronic reporting system in Lithuania. Euro Surveill. 2009:14(13):pii=19165. Available online: http://www.eurosurveillance.org/ViewArticle.aspx?ArticleId=19165 